\title{
Bilateral vocal cord palsy following hemithyroidectomy - a unique case report
}

\author{
N. Vakharia, S. Unadkat, P. Stimpson, M. Papesch \\ Whipps Cross University Hospital, Barts Health NHS Trust, London \\ Corresponding author: Nilesh.Vakharia@nhs.net
}

\section{BACKGROUND}

- Recurrent laryngeal nerve (RLN) palsy is a recognised complication of thyroid surgery, resulting in dysphonia, and if bilateral, airway compromise \& aspiration.

- RLN palsy following thyroid surgery varies, and is reported at $0.4 \%$ to $7.2 \%$ for temporary paresis and from $0 \%$ to $5.2 \%$ for permanent paralysis ${ }^{[1]}$.

- Bilateral RLN palsy as a complication of thyroid surgery is seen exclusively in total thyroidectomy, and has been described in numerous studies ${ }^{[2,3]}$.

- Following an extensive review of the literature, we present an exceptionally rare case of bilateral laryngeal nerve palsy following hemithyroidectomy, and explore the possible aetiopathogenesis.

\section{CASE}

- 36-year-old woman, one-year history of left sided neck lump.

- Palpable midline neck swelling, no sinister features

- Flexible nasolaryngoscopy (FNE): normal vocal cord movements.

- US scan: $2.2 \times 3.4 \mathrm{~cm}$ nodule within left thyroid lobe, consistent with benign nodule, graded U2 as per the BTA guidelines (2014) ${ }^{[4]}$.

- Despite small risk for malignancy, the patient opted for a hemithyroidectomy.

- Intra-operatively: ventilated using a cuffed EMG endotracheal tube, positioned supine with neck in extension. No intra-operative surgical concerns. Left recurrent laryngeal nerve identified and stimulated.

- Post-operatively: Profoundly dysphonic, but non-stridulous. Aspirating thin fluids.

\section{CASE (CONTINUED)}

- FNE: bilateral vocal cord palsy, paramedian position, suggestive of bilateral RLN paralysis.

- MRI neck (fig. 1), no abnormalities identified.

- Blood tests including corrected calcium, vitamin D and magnesium were all within the normal range.

- Management: - Oral steroids (50mg prednisolone)

- SALT assessment - thickened fluids

- Tracheostomy not required.

- Discharged day 6 post-op, outpatient follow up

- Neurology opinion - no abnormality of cranial or peripheral nervous systems, likely bilateral vocal cord dysfunction secondary to neuropraxis.

- Repeat endoscopic evaluation 10 days post-procedure: recovery of vocal cord movement on the left side, no improvement on the non-operated, right side.

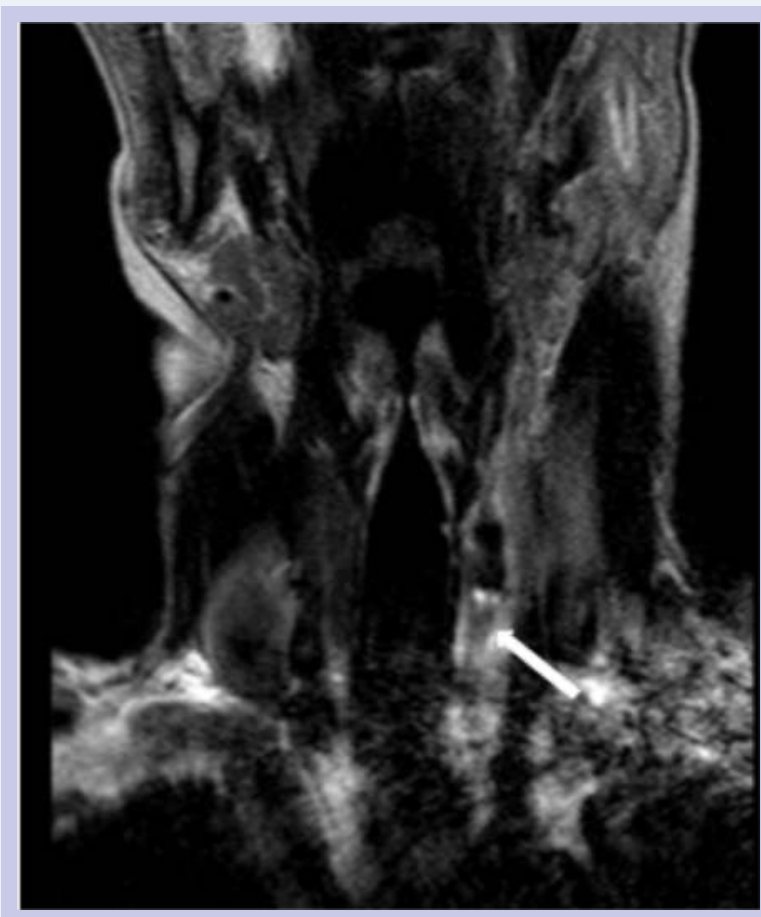

Fig. 1 - Coronal T2 sequence, left thyroidectomy (white arrow), no structural abnormality in upper mediastinum, lung apices or tracheo-oesophageal groove

- Six weeks post-op: mobility of right vocal cord, but continued impairment of vocal cord adduction. CT neck was also performed (fig. 2) at this stage to assess the subglottis, but no abnormalities were identified.

- Eight weeks post operatively: voice normal, repeat FNE: normal vocal cord movement

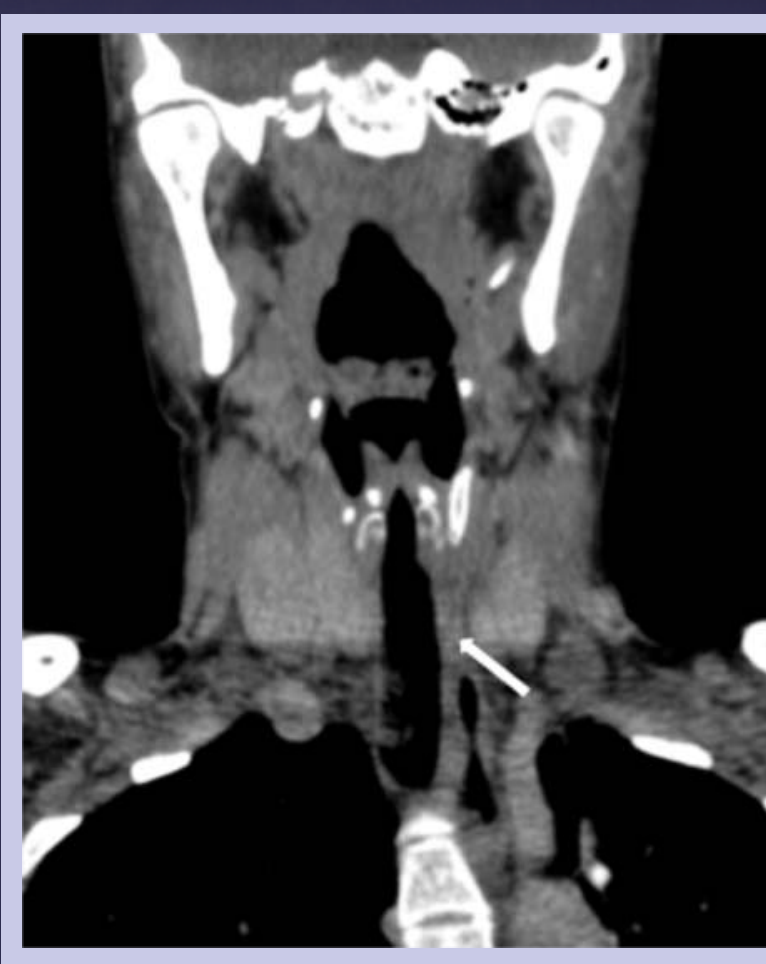

Fig. 2 - Contrast enhanced CT, no structural abnormality in upper mediastinum, lung apices or tracheo-oesophageal groove. No subglottic stenosis.

\section{DISCUSSION}

- Vocal cord dysfunction has numerous aetiologies

Non-surgical: hypocalcaemia, low vitamin D levels $s^{[5]}$, side effect of chemotherapy agents ${ }^{[6]}$.

- Hypocalcaemia due to removal of the parathyroid glands is more frequent following total thyroidectomy ${ }^{[7]}$. Compression of the RLN may occur between the endotracheal (ET) tube cuff and the rigid thyroid lamina. Pressures in ET tube cuffs inflated with air rise by $8-10 \mathrm{mmHg}$ during a procedure and may contribute to cuff-related nerve palsies ${ }^{[8]}$.

Neck hyperextension during intubation or positioning can result in stretching of the vagus nerves ${ }^{[9,10]}$.

- In our case, the patient was positioned with a shoulder roll and head ring. Neuropraxia from stretching of the nerve, compression by the ET tube cuff, or indeed a combination of both may be the cause of bilateral vocal cord paralysis seen in our case.

\section{CONCLUSION}

What is already known:

- Vocal cord paralysis is a relatively rare, but potentially devastating complication of thyroid surgery.

- Causes of vocal cord palsy may be surgical or non-surgical, and are not limited to thyroid surgery.

- Use of intra-operative nerve stimulators and loupes magnification has contributed to reducing risk of damage to the recurrent laryngeal nerve.

What this case adds:

- A unique case of bilateral vocal cord palsy following hemithyroidectomy surgery

- A review of the aetiopathogenesis of vocal cord palsy, and the steps that can be taken during surgical procedures to prevent it.

\section{REFERENCES}

Rulli F, Ambrogi V, Dionigi $G$ et al. Meta-analysis of recurrent laryngeal nerve injury in thyroid surgery with 6 or without intraoperative nerve monitoring. 2014;73:223-9.

Dy AES. Transient bilateral vocal fold paralysis after total thyroidectomy. Turkish J. Ear Nose Throat 2016;26(6):356-9.

Rangappa VB, Ramakrishnan A, Balasubramanya AM, Ram R. Bilateral Adductor Palsy : A Rare Complication during Routine Surgery. :76-8.

4. Perros P, Colley S, Boelaert K et al. Guidelines for the management of thyroid cancer. Clin. Endocrinol. (Oxf). 2014;81(Supplement 1 July 2014):1-136.

Büyükcam F, Sönmez FT, Şahinli H. A delayed diagnosis: Stridor secondary to hypocalcemia. Int. J. Emerg.
Med. 2010;3(4):461-2

Myssiorek D. Recurrent laryngeal nerve paralysis: Anatomy and etiology. Otolaryngol. Clin. North Am. 2004;37(1):25-44

. Bourrel C, Uzzan B, Tison P et al. Transient Hypocalcemia after Thyroidectomy. Ann. Otol. Rhinol. Laryngol. 1993;102(7):496-501.

8. Cavo JW. True vocal cord paralysis following intubation. Laryngoscope1985;95(11):1352-9.

9. Nuutinen J, Kärjä J. Bilateral vocal cord paralysis following general anesthesia. Laryngoscope 1981;91(1):83-6.

10. Vyshnavi S, Kotekar N. Aphonia following tracheal intubation: An unanticipated post-operative complication. Indian J. Anaesth.2013;57(3):306-8. 